\title{
Measurement of Thermo-Optic Coefficient of a Liquid Using a Cascade of Two Different Fiber Bragg Gratings
}

\author{
Kwang Taek Kim ${ }^{+}$and In Soo Kim
}

\begin{abstract}
We proposed and demonstrated a fiber optic sensor for detecting the thermo-optic coefficient of a liquid, based on a cascade of two different FBGs. One of the two FBGs was etched, and its cladding was removed, for evanescent wave coupling with an external liquid. The Bragg wavelength of the non-etched FBG was used as a reference for the temperature of the surrounding liquid. The refractive index (RI) and thermo-optic (T-O) coefficient of a liquid can be detected from the difference between the Bragg wavelengths of the two FBGs, and the variation of the difference in accordance with temperature.
\end{abstract}

Keywords : Fiber Bragg grating, Temperature sensor, Refractive index sensor, Thermo-optic coefficient

\section{INTRODUCTION}

Optical fiber sensors have attractive features, including the capability of remote and distributed sensing, immunity to electromagnetic interference, wavelength multiplexing, and durability in aggressive environments, high sensitivity, fast response, light weight and small size. The simultaneous measurement of the refractive index (RI) is one of the most important issues. In the past years, various fiber optical sensing techniques have been developed for measurement of RI using a microring resonator [1], MachZehnder interferometer [2], long period grating [3, 4] and FBG (Fiber Bragg Grating) [5-7]. The T-O coefficient which means change of RI per variation of unit temperature is one of the important parameters determining the optical property of a medium, along with the RI. It is well known that the Bragg wavelength of cladding etched FBGs is sensitive to the RI of the surrounding material [8, 9] because the modal characteristics of FBG are affected by the liquid, through evanescent wave coupling. In order to obtain the T-O coefficient of a medium, the RI and temperature should be measured simultaneously. However,

Department of Opto-electronics, Honam Unvinersity, 59-1, Seobong Dong, Gwangsan Gu, Gwangju, Korea,

${ }^{+}$Corresponding author: ktkim@honam.ac.kr

(Received : Feb. 4, 2013, Accepted : Feb. 24, 2013)

This is an Open Access article distributed under the terms of the Creative Commons Attribution Non-Commercial License(http://creativecommons.org/licenses/bync/3.0)which permits unrestricted non-commercial use, distribution, and reproduction in any medium, provided the original work is properly cited. it is difficult to simultaneously measure the RI and temperature of a liquid using only a cladding etched FBG, because of the cross sensitivity between those two parameters. It has been reported that the partially cladding removed FBG can be used for simultaneous measurement of the two parameters $[9,10]$.

In this paper, we have investigated a measurement method to determine the thermo-optic (T-O) coefficient, using a combination of two different FBGs. Naturally, the proposed sensor can be used for simultaneous measurement of temperature and the RI of a liquid. The cladding of one FBG is removed by wet etching. The temperature can be obtained from the Bragg wavelength of the non-etched FBG, whereas the RI of the external medium can be acquired from the difference in Bragg wavelength between the two FBGs. A graphical approach to determine the T-O coefficient of the liquid is presented. Several materials were examined, and the validity of the proposed method was experimentally proved.

\section{THEORETICAL CONSIDERATION}

The proposed fiber-optic sensor is composed of two adjacent FBGs with different Bragg wavelengths, as shown in Fig. 1. The cladding of the second FBG is removed by chemical etching, so that the Bragg wavelength of the FBG is sensitive to the RI of the external medium, due to evanescent wave coupling. The Bragg wavelength of an FBG made of a single mode optical fiber is given by 


$$
\lambda_{B}=2 n_{e f f} \Lambda
$$

Here, the grating period $(\Lambda)$ and effective RI $\left(n_{\text {eff }}\right)$ of the single-mode fiber core depend on the temperature applied to the fiber.

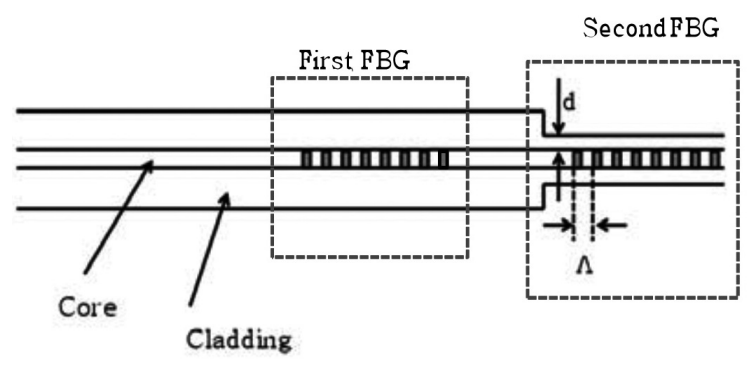

Fig.1. Schematic structure of FBG sensor for measurement of T-O coefficient.

Fig. 2 shows the effective RI according to the RI of an external medium, for several different remaining cladding thicknesses (d). A standard single-mode fiber (SMF 28) with a core diameter of $8.2 \mu \mathrm{m}$ is considered. In the calculation, the RIs of the core and cladding are 1.4492 and 1.4440 , at $1550 \mathrm{~nm}$ wavelength, respectively. The grating period $(\Lambda)$ is $0.53414 \mu \mathrm{m}$. As the RI of the external medium $\left(\mathrm{n}_{\mathrm{ex}}\right)$ decreased, the effective RI of the fiber mode also decreased. The relation between the two parameters is nonlinear as shown in Fig. 2. Once the RI of an external medium is given, it is also possible to estimate the remaining cladding (d) thickness using the Bragg wavelength difference in regard to before and after cladding etching.

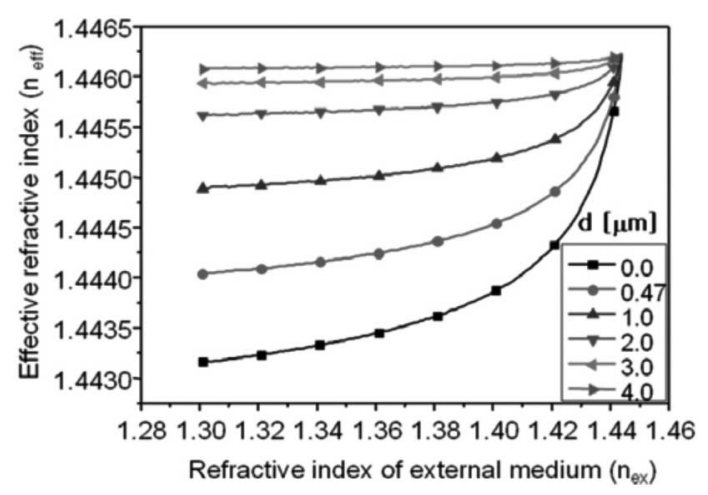

Fig. 2. Effective RI of cladding etched single mode fiber in accordance with RI of an external medium for several different remaining cladding thicknesses (d).
It is known that the Bragg wavelength of FBG is shifted in accordance with temperature due to the $95 \%$ thermooptic effect and 5\% thermo-expansion effect [11]. The temperature sensitivity of the typical FBG is known to be approximately $1.1 \mathrm{~nm} /{ }^{\circ} \mathrm{C}[10]$. Therefore, the Bragg wavelength of the first FBG depicted in Fig. 1 is only dependent on temperature, and can be used for temperature monitoring. The RI of liquids is acquired using the difference between the Bragg wavelengths of the two FBGs $[8,9]$. If the thermo-optic effect of the external medium is negative, the decrease of $n_{e x}$ with increase of temperature of the liquid results in self-compensation of the positive thermo-optic effects [10]. The change of Bragg wavelength for cladding removed FBG (second FBG) is caused by both the thermal effect of silica fiber and T-O effect of the surrounding liquid. Therefore, the change in effective RI and shift of Bragg wavelength regarding FBG is expressed as [12] :

$$
\Delta \lambda_{B} \cong \Delta \lambda_{B}^{F}+\Delta \lambda_{B}^{L}=2 \Lambda\left(\Delta n_{e f f}^{F}+\Delta n_{e f f}^{L}\right)
$$

Here, $\Delta \lambda_{B}^{F}$, and $\Delta \lambda_{B}^{L}$, denote change in the Bragg wavelength by the thermal effect of the fiber itself and the surrounding liquid, respectively. $\Delta n_{\text {eff }}^{F}$ and $\Delta n_{e f f}^{L}$ are the corresponding variation of the effective RI contributed by the fiber itself and the surrounding liquid, respectively. If the thermal characteristics of FBG are not changed after cladding etching, $\Delta \lambda_{B}^{F}$ of the second FBG can be obtained from the Bragg wavelength shift of the first FBG. Since $\Delta \lambda_{B}$ of the second FBG can be measured experimentally, we can use $\Delta \lambda_{B}^{L}=\Delta \lambda_{B}-\Delta \lambda_{B}^{F}$. Once the remaining cladding thickness and grating period of the cladding etched FBG are given, the change in RI of the external medium can be obtained from the $\Delta \lambda_{B}^{L}$. Therefore, we can obtain the T-O coefficient defined as $\frac{\Delta n_{e x}}{\Delta T}$.

\section{EXPERIMETAL RESULTS AND ANALYSIS}

Two Bragg gratings were inscribed in a single mode fiber, using the general phase mask method. The gap between the first FBG and second FBG is $5.0 \mathrm{~mm}$. Bragg wavelengths of the first and second gratings were designed to be $1550 \mathrm{~nm}$ and $1545 \mathrm{~nm}$ at $22^{\circ} \mathrm{C}$. Although Bragg wavelengths of two the FBGs are slightly different, their thermal characteristics are assumed to be equal. The 
second FBG fiber was immersed and chemically etched in an aqueous solution of hydrofluoric acid (HF 40\%) at $60^{\circ} \mathrm{C}$. With an approximate etching rate of $1.0 \mu \mathrm{m} / \mathrm{min}$, the fiber cladding was etched close to the core of the fiber.

Fig. 3 presents the experimental setup for measuring the sensing characteristic of FBG sensors. The reflection spectrum of the sensors was monitored using an OSA (Optical Spectrum Analyzer).

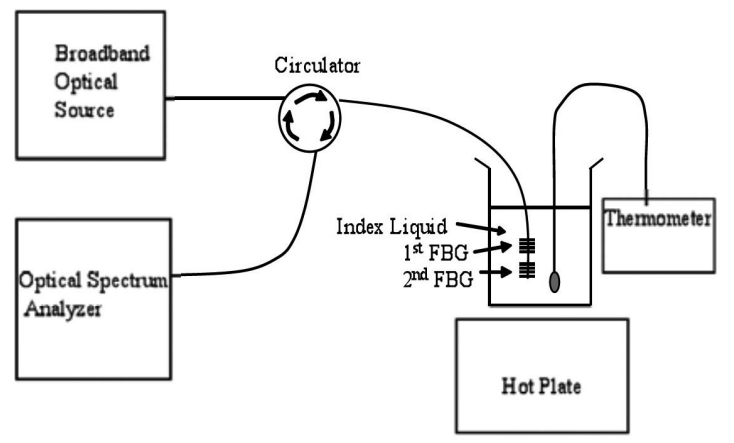

Fig. 3. Experimental setup for measuring of reflection spectrum of FBG sensors.

Fig. 4 shows the measured reflection spectrum before and after cladding etching under air circumstance, i.e. $n_{\mathrm{ex}}$ $=1.0$. The shifting of Bragg wavelengths of the second FBG after etching was $2.66 \mathrm{~nm}$, respectively. The estimated remaining cladding thickness (d) after etching was $0.47 \mu \mathrm{m}$.

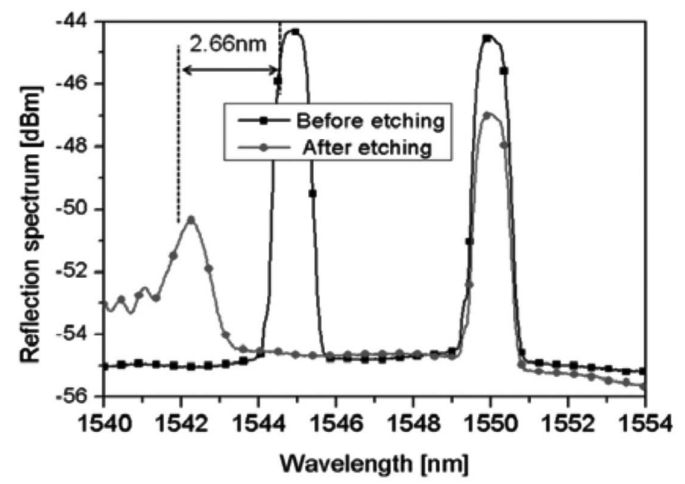

Fig. 4. Measured reflection spectrum of the FBG sensor before and after cladding etching.

We measured the influence of the RI of a liquid surrounding both FBGs, on the reflection spectrum under a constant temperature of $22^{\circ} \mathrm{C}$. For this experiment, several liquids made of the water-glycerin mixture with different RI were prepared. The RIs of liquids were adjusted by control of the mixing ratio regarding the two media, and were measured using a prism coupler (SPA 4000, Sairon Korea) at a wavelength of $1550 \mathrm{~nm}$. The Bragg wavelength of the first FBG was almost fixed, regardless of the value of $n_{e x}$, while that of the second FBG is sensitive to $n_{e x}$, as shown in Fig. 5. As $n_{e x}$ increased, the Bragg wavelength of the second FBG shifted to the longer wavelength region.

Fig. 6 shows the measured Bragg wavelength, in accordance with the RI of liquids. The experimental results are in good agreement with the theoretical fit. Here, the remaining cladding thickness is assumed to be $0.47 \mu \mathrm{m}$.

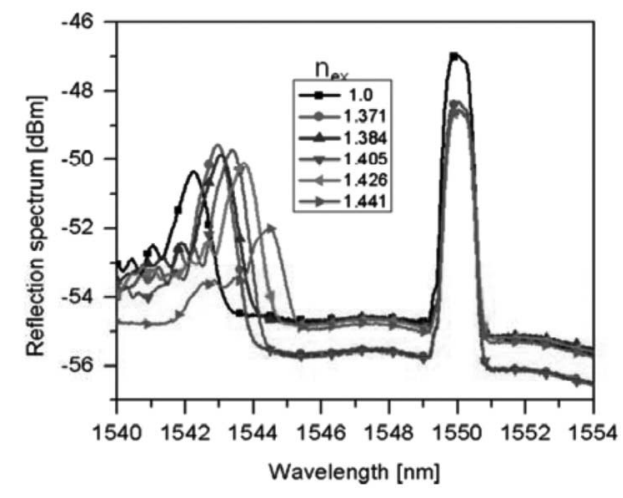

Fig. 5. Measured reflection spectrum for several different external mediums with different RIs.

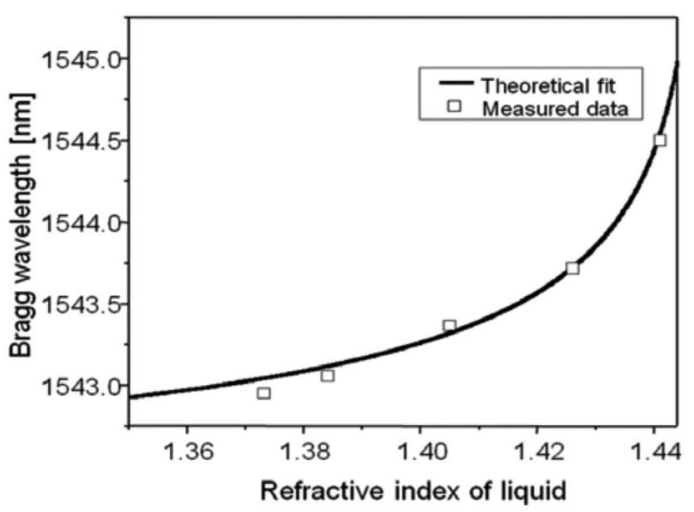

Fig. 6. Measured Bragg wavelength for several liquids with different RIs.

We measured the thermal properties of the proposed sensor when both FBGs were surrounded by a liquid. The experimental results are shown in Fig. 7. The temperature sensitivity of the first FBG is approximately $0.011 \mathrm{~nm} /{ }^{\circ} \mathrm{C}$, and its value is almost equal to a previous report [1]. In this experiment, the first FBG is used as a temperature sensor. When the cladding removed FBG (second FBG) is surrounded by air, its temperature sensitivity is almost 
equal to that of the first FBG, as shown in Fig. 7 (a). This means that the thermal characteristics of FBGs are not changed after the cladding is removed. When the liquids, water, glycerin $(80 \mathrm{wt} \%)$, and glycerin $(90 \mathrm{wt} \%$ ) were used as the external medium, the shifts of Bragg wavelength for the second FBG were smaller than those of the first FBG.

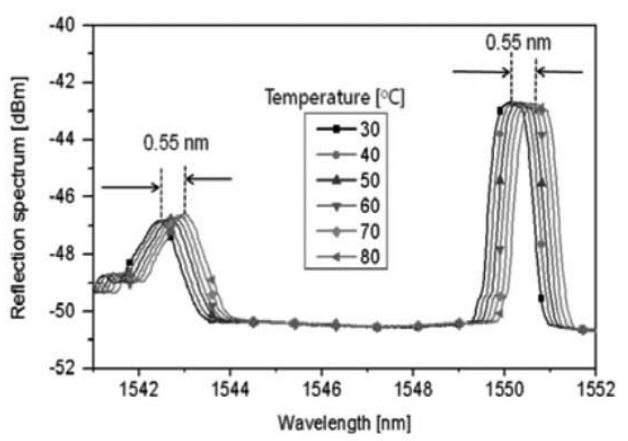

(a)

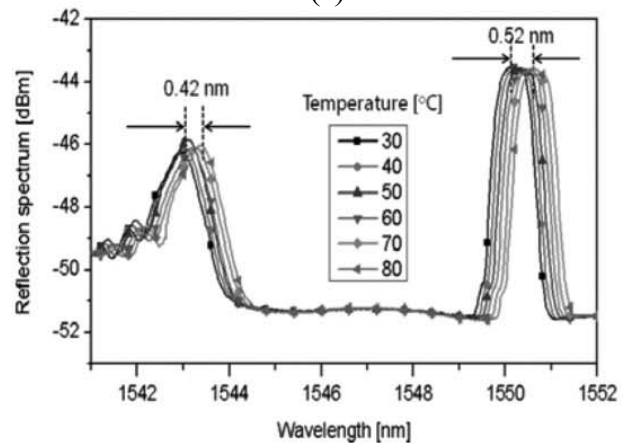

(b)

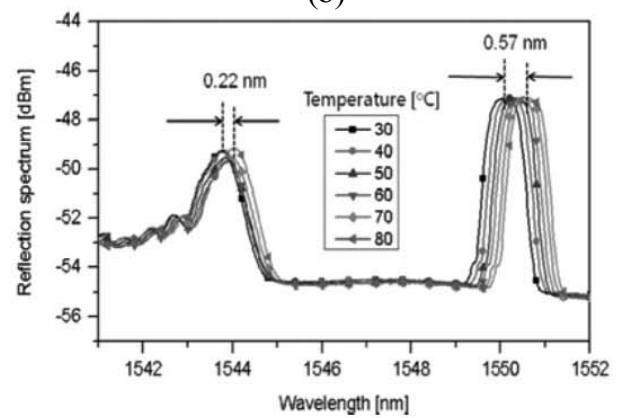

(c)

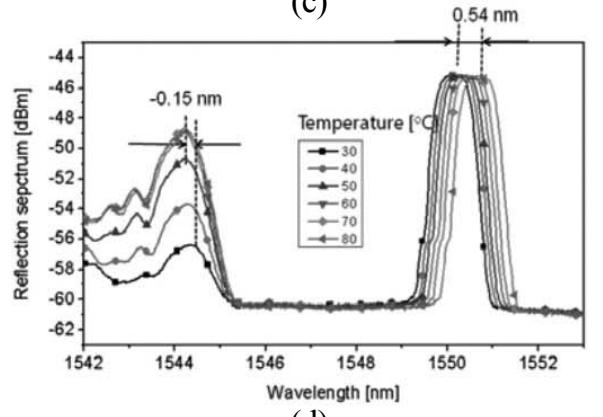

(d)

Fig. 7. Measured refraction spectra with variation of temperature for different external media; (a) air, (b) pure water, (c) glycerin $(80 \% \mathrm{wt})$, and (d) glycerin $(90 \% \mathrm{wt})$.
This phenomenon is the result of the negative thermooptic coefficient of liquids. Using the Bragg wavelength shift of the two FBGs and the difference of two Bragg wavelengths, the T-O coefficient of the liquid is determined. Fig. 8 explains how we determine the T-O optic coefficient of the pure water depicted in Fig. 7 (b). When the temperature of the water was increased from 30 to $40{ }^{\circ} \mathrm{C}$, the measured $\Delta \lambda_{B}^{F}$ of the first FBG and $\Delta \lambda_{B}$ of the second FBG were $+0.011 \mathrm{~nm}$ and $+0.009 \mathrm{~nm}$. Therefore, $\Delta \lambda_{B}^{L}$ was $-0.002 \mathrm{~nm}$. This corresponds to a RI change of 0.008 of the liquid during a change of $10^{\circ} \mathrm{C}$. Therefore, the T-O coefficient of pure water was $-0.8 \times 10^{-4}$ ${ }^{\circ} \mathrm{C}$. This result is well matched with previously reported works $[8,12]$. In this way, we could find that the T-O coefficients of glycerin (90 wt $\%$ ) depicted in Fig. 7(d) was $-2.9 \times 10^{-4} /{ }^{\circ} \mathrm{C}$.

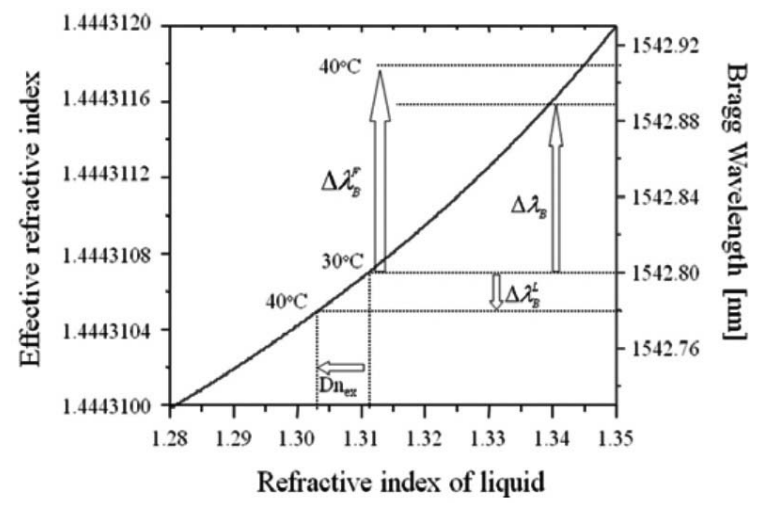

Fig. 8. Graphical approach to obtain the T-O coefficient of pure water using the Bragg wavelength shift in accordance with temperature.

In this paper, we have investigated the thermal characteristic of the cascaded cladding etched FBG and non-etched FBG, to detect the T-O coefficients of a liquid. A simple graphical approach is presented to acquire the T$\mathrm{O}$ coefficient. The validity of the proposed methods was experimentally demonstrated. Naturally, the sensor can be used for simultaneous measurement of temperature and RI.

\section{CONCLUSIONS}

We have studied a measurement method for the T-O coefficient of a liquid, based on a combination of two different FBGs. One of FBGs was wet etched and its cladding was removed to obtain evanescent wave sensing. The remaining cladding thickness after wet etching was 
estimated by the Bragg wavelength shift before and after cladding etching. The Bragg wavelength of non-etched FBG was only influenced by the temperature of the liquid while the cladding etched FBG was affected by both temperature and the RI of the liquid. The measured RIs of liquids using the proposed method were well matched with the results obtained by the prism coupler. We found that the temperature sensitivity of the Bragg wavelength for the cladding removed is almost equal to that of the standard FBG when the etched region was surrounded with air. However, when the cladding removed FBG was surrounded with a liquid its Bragg wavelength sensitivity to temperature was smaller than that of the non-etched FBG, because of the negative T-O coefficient of the liquid. We have presented a simple graphical method to obtain the $\mathrm{T}-\mathrm{O}$ coefficient of a liquid using the Bragg wavelength shift of two FBGs in accordance with temperature, and the experimental results demonstrated the validity of the proposed method.

\section{REFERENCES}

[1] N. Lin, L. Jiang, S. Wang, L. Yuan, and Q. Chen, "Simultaneous measurement of refractive index and temperature using a microring resonator", Chin. Opt. Lett., Vol. 10, No. 5, pp. 52802-52900, 2012.

[2] L. Simultaneous measurement of refractive index and temperature using thinned fiber based Mach-Zehnder interferometerLi, X. Li, Z. Xie, Z. Liao, F. Tu, and D. Liu, "Simultaneous measurement of refractive index and temperature using thinned fiber based MachZehnder interferometer", Opt. Commun., Vol. 285, No. 10, pp. 3945-3949, 2012.

[3] V. Bhatia, D. Campbell, R. O. Claus, and A. M. Vengsarkar, "Simultaneous strain and temperature measurement with long-period gratings", Opt. Lett., Vol. 22, No. 9, pp. 648-650, 1997.

[4] Y.-J. Rao and Q.-J. Mo, "Simultaneous measurement of refractive index and temperature using a single ultralong period fiber grating," IEEE Photonic Technol. Lett., Vol. 17, No. 12, pp. 2700-2702, 2005.

[5] C.-L. Zhao, X. Yang, and M.S. Demokan, "Simultaneous temperature and refractive index measurements using a $3^{\circ}$ slanted multimode fiber Bragg grating", J. Lightwave Technol., Vol. 24, No. 2, pp. 879-883, 2006.

[6] X. Shu, B.A.L. Gwandu, Y. Liu, L. Zhang, and I. Bennion, "Sampled fiber Bragg grating for simultaneous refractive-index and temperature measurement”, Opt. Lett., Vol. 26, No. 11, pp. 774-776, 2001.

[7] Z. Chen, J. Tang, R. Fan, Y. Zhong, J. Zhang, and S. Li, "Side-polished fiber Bragg grating sensor for simultaneous measurement of refractive index and temperature", 21st International Conference on Optical Fiber Sensors, p.7753, 2011.

[8] A. Iadicicco, S. Campopiano, A. Cutolo, M. Giordano, and A. Cusano, "Self temperature referenced refractive index sensor by non-uniform thinned fiber Bragg gratings", Sens. Actuator A-Phys., Vol. 120, No. 1, pp. 231-237, 2006.

[9] X. Sang, C. Yu, T. Mayteevarunyoo, K. Wang, Q. Zhang, and P. L. Chu, "Temperature-insensitive chemical sensor based on a fiber Bragg grating", Sens. Actuator A-Phys., Vol. 120, No. 2, pp. 754-757, 2007.

[10] A. D. Kersey, M. A. Davis, H. J. Petrick, M. LeBlanc, K. P. Koo, C. G. Askins, M. A. Putnam, and E. J. Friebele, "Fiber grating sensors", J. Lightwave Technol., Vol. 15, pp. 1442-1463, 1997.

[11] R. C. Kamikawachi, I. Abe, A. S. Paterno, H. J. Kalinowski, M. Muller, J. L. Pinto, and J. L. Fabris, "Determination of thermo-optic coefficient in liquids with fiber Bragg grating refractometer", Opt. Commun., Vol. 281, No. 4, pp. 621-625, 2008.

[12] A. N. Chryssis, S. S. Saini, S. M. Lee, and M. Dagenais "Increased sensitivity and parametric discrimination using higher order modes of etchedcore fiber Bragg grating sensors", IEEE Photonic Technol. Lett., Vol. 18, No. 3, pp. 178-180, 2006. 